\title{
Sleep apnoea and the hypothalamic- pituitary-adrenal axis in men and women: effects of continuous positive airway pressure
}

\author{
Ilia Kritikou', Maria Basta ${ }^{1,2}$, Alexandros N. Vgontzas' ${ }^{1}$, Slobodanka Pejovic ${ }^{1}$, \\ Julio Fernandez-Mendoza ${ }^{1}$, Duanping Liao ${ }^{3}$, Edward O. Bixler ${ }^{1}$, Jordan Gaines ${ }^{1}$ \\ and George P. Chrousos ${ }^{4}$
}

Affiliations: ${ }^{1}$ Sleep Research and Treatment Center, Dept of Psychiatry, Pennsylvania State University, College of Medicine, Hershey, PA, USA. ${ }^{2}$ Dept of Psychiatry, University of Crete School of Medicine, Heraklion, Greece. ${ }^{3}$ Dept of Public Health Sciences, Pennsylvania State University, College of Medicine, Hershey, PA, USA. ${ }^{4}$ First Dept of Pediatrics, National and Kapodistrian University of Athens School of Medicine, Athens, Greece.

Correspondence: Alexandros N. Vgontzas, Pennsylvania State University College of Medicine, Dept of Psychiatry H073, 500 University Drive, Hershey, PA 17033, USA. E-mail: avgontzasahmc.psu.edu

ABSTRACT Previous findings on the association of obstructive sleep apnoea (OSA) and the hypothalamic-pituitary-adrenal (HPA) axis are inconsistent, partly due to the confounding effect of obesity and infrequent sampling. Our goal was to examine whether in a relatively nonobese population, OSA is associated with elevated cortisol levels and to assess the effects of a 2-month placebo-controlled continuous positive airway pressure (sham-CPAP) use.

72 subjects ( 35 middle-aged males and post-menopausal females with OSA, and 37 male and female controls) were studied in the sleep laboratory for four nights. 24-h blood sampling was performed every hour on the fourth day and night in the sleep laboratory at baseline, after sham-CPAP and after CPAP treatment.

In both apnoeic men and women, OSA was associated with significantly higher 24-h cortisol levels compared with controls, whereas CPAP lowered cortisol levels significantly, close to those of controls.

These results suggest that OSA in nonobese men and slightly obese women is associated with HPA axis activation, similar albeit stronger compared with obese individuals with sleep apnoea. Short-term CPAP use decreased cortisol levels significantly compared with baseline, indicating that CPAP may have a protective effect against comorbidities frequently associated with chronic activation of the HPA axis, e.g. hypertension.

@ERSpublications

OSA in nonobese men/slightly obese women is associated with HPA axis activation and CPAP lowers HPA axis activity http://ow.ly/Stn94

For editorial comment see Eur Respir J 2016; 47: 366-368 [DOI: 10.1183/13993003.01920-2015].

Received: Feb 252015 | Accepted after revision: Sept 082015 | First published online: Nov 052015

Conflict of interest: Disclosures can be found alongside the online version of this article at erj.ersjournals.com

Support statement: This study was supported by the US Department of Health and Human Services, National Institutes of Health. Funding information for this article has been deposited with FundRef.

Copyright OERS 2016 


\section{Introduction}

Obstructive sleep apnoea/hypopnea syndrome (OSA) is characterised by repetitive episodes of upper airway obstruction leading to complete airflow cessation (apnoeas) or partial airflow reduction (hypopneas) that result in intermittent hypoxaemia, electroencephalographic arousals and sleep fragmentation [1]. The associated hypoxaemia and the concomitant increase in arterial carbon dioxide tension are expected to result in sympathetic activation and catecholamine secretion, while the repeated arousals can cause activation of the hypothalamic-pituitary-adrenal (HPA) axis, thus increasing cortisol release [2]. However, and contrary to what was expected, most studies have reported no significant association [3-8] or decreased HPA axis activity [9], whereas only two studies reported increased HPA axis activity in sleep apnoea patients compared with controls [10, 11]. A more recent study [12] found a significant association between apnoea severity and 24-h cortisol values; however, it was limited by the absence of a control group. Methodological limitations such as single blood draws, lack of a control group, failure to match comparison groups (e.g. by age and body mass index (BMI)) and inadequate control of variables known to affect HPA function may be the underlying factors of these inconsistent findings [13].

Furthermore, studies on the association between cortisol levels and sleep apnoea have focused mainly on obese middle-aged male populations, whereas data in nonobese men as well as women are lacking [13]. Obesity is the strongest risk factor for sleep apnoea, but also a known HPA axis modulator, and thus a possible confounder of the association between sleep apnoea and HPA axis activity [11].

Given the above limitations, it is no surprise that studies on the effects of continuous positive airway pressure (CPAP) treatment on HPA axis activity have yielded inconsistent findings [8, 10, 11, 13-17]. In addition, most of the CPAP studies have been confounded by methodological limitations such as lack of a control group at baseline, or a placebo control group, as well as a small sample size. From the available published studies, only one has included a placebo-controlled arm, but this study was limited by a single morning blood draw [16].

To address these gaps in the literature we assessed in a carefully characterised population of sleep apnoeics and controls the basal cortisol secretory pattern using hourly 24-h serial blood sampling in a predominantly nonobese population of apnoeic men and women, and their respective controls, and examined the independent and gender-specific association with OSA. Despite our efforts, our sample of women consisted of slightly obese patients because 1) in central Pennsylvania women are heavier compared with national standards and 2) women with OSA are heavier than men, both in clinical and epidemiological samples $[18,19]$. A secondary goal was to assess the effect of a placebo-controlled (sham-CPAP) 2-month CPAP trial on the circadian cortisol secretory profile.

\section{Materials and methods}

Subjects

In total, 72 subjects ( 36 males, 36 females), including 35 sleep apnoea patients (19 males, 16 females) and 37 controls (20 males, 17 females), participated in this study for the assessment of the 24 -h circadian cortisol secretory pattern in sleep apnoea in a population of nonobese middle-aged men and slightly obese middle-aged post-menopausal women (menopause was diagnosed based on the history given by the participants).

The subjects were recruited from the sleep disorders clinic and through advertisements from the community. To qualify for the study, apnoeic patients had to have apnoea of sufficient severity to warrant recommendation for treatment. We selected more stringent criteria than those currently recommended by sleep organisations and insurance companies, because we wanted to examine patients with the more severe disease. These criteria included an apnoea-hypopnea index (AHI) of $>10$ events $\cdot \mathrm{h}^{-1}$ of sleep for females and $>15$ events $\cdot \mathrm{h}^{-1}$ for males, because females have on average lower indices of respiratory disturbance and tend to manifest symptoms at a lower threshold [18]. A thorough medical assessment, including physical examination, routine laboratory tests (including complete blood cell count, urinalysis, kidney function, fasting lipid profile, thyroid function tests, electrocardiography and urine drug screen) and sleep history, was completed for each patient and control subject. Those who were positive for abnormal findings in the battery of clinical tests (apart from the fasting lipid profile) were excluded from the study.

Exclusion criteria included a history of diabetes mellitus type 2, the use of antiglycaemic agents or fasting glucose blood levels $>126 \mathrm{mg} \cdot \mathrm{dL}^{-1}$ at the time of screening, ongoing infections, rheumatoid arthritis, insomnia, narcolepsy, and use of specific medications (psychotropics, steroids, sympathomimetics or sympatholytics, or hormone therapy for females). Subjects with extreme sleep schedules, employed in shift work or with a primary circadian disorder were also excluded from the study.

Sleep apnoea patients that had used CPAP previously were excluded from the study. 
The study was approved by the University Institutional Review Board and all participants provided written informed consent.

\section{Procedures}

\section{Sleep laboratory}

A thorough medical assessment, including history, physical examination, routine laboratory tests and sleep history, was completed for each participant as described in more detail above [18, 19]. Anthropometric parameters were obtained and BMI was calculated based on height and weight measured as part of the physical examination. All potential participants were screened in the sleep laboratory for one night for $8 \mathrm{~h}$; subjects who met the inclusion criteria were then monitored in the sleep laboratory for four consecutive nights (one adaptation and three baseline nights) $[18,19]$. For the sleep monitoring, all of the subjects slept in a sound-attenuated, temperature- and light-controlled room in our sleep laboratory. During this time, the subject's sleep was continuously monitored for $8 \mathrm{~h}$ (24-analogue-channel and 10-DC-channel Aurora TS amplifier system using Gamma software; Grass-Telefactor, West Warwick, RI, USA). A four-channel electroencephalogram, a two-channel electro-oculogram and a single-channel electromyogram were recorded. The sleep records were subsequently scored independently according to standardised criteria. Respiration was monitored throughout the night by use of thermocouple at the nose and mouth (Pro-Tech, Murrysville, PA, USA), nasal pressure (MP $45 \pm 2 \mathrm{cmH}_{2} \mathrm{O}$; Validyne Engineering, Northridge, CA, USA), and thoracic and abdominal strain gauges (model 1312; Sleepmate Technologies; Midlothian, VA, USA). We obtained an objective estimate of snoring during the polysomnography by monitoring breathing sounds with a microphone attached to the throat (model 1250; Sleepmate Technologies), as well as a separate room microphone. A single-channel ECG was also recorded. All-night haemoglobin oxygen saturation was obtained from the finger (model 8600; Nonin Medical; Plymouth, MN, USA).

During the duration of the study, patients were asked to maintain their daily routine programme, calorie/ fat intake and level of physical activity steady. Due to the study design, dietary habits and caloric intake/ energy expenditure were not assessed on a daily basis, as this was not feasible. During the stay in the sleep laboratory, subjects were instructed not to change their diet, and their three daily meals were at about 07:00, 12:00 and 18:00 h. Diet composition was similar for control and apnoeic volunteers while in the sleep laboratory.

The study design included also two consecutive 2-month periods of CPAP and sham-CPAP treatment for OSA patients in a random counterbalanced order as described previously [18]. Assignment was done to an 1:1 ratio randomly, since with the current $2 \times 2$ crossover design, each subject serves as a control for him/ herself. Technical/medical staff and patients were blinded to the treatment they were getting; patients were reassessed with the same protocol as previously described at the end of each 2-month period. The sleep records were scored independently of any knowledge of the experimental conditions according to standardised criteria [18]. A flowchart of the study is given in figure 1.

\section{4-h blood sampling}

24-h serial blood samples were collected, every $60 \mathrm{~min}$ on the fourth day and night in the sleep laboratory at baseline, and repeated in sleep apnoea patients at the end of both CPAP and sham-CPAP treatment periods. During the sleep periods, blood samples were obtained from an adjacent room by connecting external tubing to the indwelling catheter through a perforation in the wall.

Hormonal assays

Blood collected from the indwelling catheter was transferred to an EDTA-containing tube and refrigerated until centrifugation (within $3 \mathrm{~h}$ ). The plasma was frozen at $-20{ }^{\circ} \mathrm{C}$ until assaying. Cortisol levels were measured by specific immunoassay techniques as previously described [11] using the Coat-A-Count Cortisol assay (Siemens, Tarrytown, NY, USA). The lower limit of detection for cortisol was $0.7 \mu \mathrm{g} \cdot \mathrm{dL}^{-1}$, and the intra- and interassay coefficients of variation were $4.3 \%$ and $5.2 \%$, respectively.

Anxiety and depression symptoms

Anxiety and depression symptoms were assessed with the Beck Anxiety Inventory (BAI) and Beck Depression Inventory II (BDI-II), respectively.

CPAP and sham-CPAP usage

All patients with sleep apnoea underwent consecutively CPAP and sham-CPAP treatment in a random counterbalanced order. The optimal nasal CPAP pressure was determined during a full-night polysomnographic study as the pressure necessary to abolish all respiratory events and snoring, secondary arousals and episodes of arterial oxygen saturation $\left(\mathrm{SaO}_{2}\right)$ desaturation during REM sleep and in the supine position. During the sham-CPAP phase, patients were given an identical machine (S8 Escape; ResMed, San 


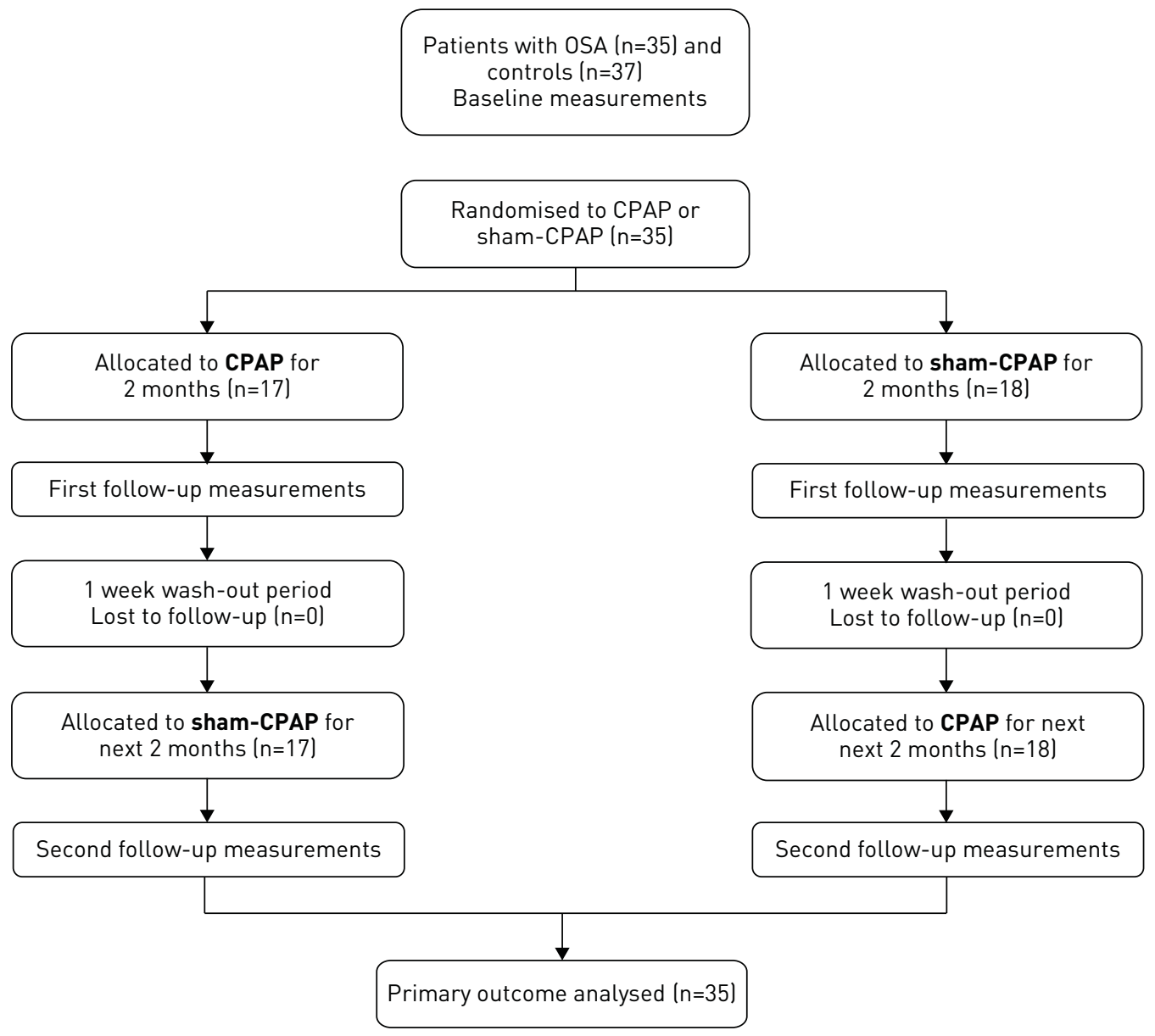

FIGURE 1 Flowchart of study subjects. OSA: obstructive sleep apnoea; CPAP: continuous positive airway pressure.

Diego, CA, USA), but the pressure derived was set at the level of $1 \mathrm{~cm} \mathrm{H}_{2} \mathrm{O}$ when used with a modified mask. The mask (Ultra Mirage II nasal mask; ResMed) was modified by adding an airflow restrictor and by increasing the amount of exhalation ports. Moreover, the airflow through the exhalation port and the operating noise in the sham-CPAP were virtually identical to those of active CPAP, which allowed successful replication. To ensure CPAP adherence, CPAP usage was monitored closely on a daily basis by calculating the time the patient was breathing through the machine and not just the time the machine was on. We accomplished that because the CPAP that we used, AutoSet T, has a function called SmartStart. Based on this function, AutoSet T starts automatically when the patient breathes into the mask and stops automatically when the patient takes the mask off. A respiratory therapist visited the home of each patient regularly to provide us with information regarding CPAP usage (number of hours used daily, pressure settings and mask leakage) and reinforce adherence. The respiratory therapist continued to visit patients following the same protocol during the sham-CPAP phase. Regular users of CPAP were considered as those who used the apparatus for at least $4 \mathrm{~h}$ each night, five or more nights per week. The design of the study also included an intervening 1-week wash-out period between the two treatment phases. Patients, investigators and respiratory therapists were all blinded to treatment phase.

\section{Statistical analysis}

For comparisons of the groups' anthropometric baseline characteristics, the independent-samples t-test was used and data are reported as mean \pm SD. The Kolmogorov-Smirnov test was used to confirm normality. Variables not normally distributed (e.g. AHI, BDI-II and BAI scores) were logarithmically transformed in the analyses. Sleep variables were calculated based on the mean values from nights 2 and 3 (night 4 was not included due to potential blood-draw-induced sleep disturbance) and were compared using the independent-samples t-test. These data are reported as mean \pm SD.

At baseline, comparisons of the 24-h cortisol values between control and apnoeic subjects were conducted using linear mixed-effects models for repeated measures after including group, gender, time and their 
interaction as fixed effects, and age, BMI, anxiety, depression and smoking as covariates, assuming the within-subjects observations followed a group-specific autoregressive variance-covariance structure (AR(1)).

For comparisons between baseline and post-CPAP and sham-CPAP phases, linear mixed-effects models were used for the repeated-measures analyses. Again, the specified covariance structure was $\operatorname{AR}(1)$. In the case of cortisol, group (baseline, sham-CPAP, CPAP), gender and time as well as their interactions were treated as fixed effects. BMI, anxiety, depression, order of intervention (sham-CPAP first or CPAP first) as well as apnoea severity (mild/moderate versus severe using a cut-off of AHI=30 events $\cdot \mathrm{h}^{-1}$ ) were also added as fixed factors in the model. The results were analysed for the entire $24-\mathrm{h}$ period, daytime (08:00-22:00 h) and night-time (23:00-07:00 h) periods.

$\mathrm{p}<0.05$ was used to determine statistical significance. Data compared with linear mixed-effects models are reported as estimated mean \pm SE. Analyses were conducted using SAS version 9.3 (SAS, Cary, NC, USA).

\section{Results}

Demographic, sleep, respiratory and emotional distress data

Demographic and sleep characteristics of the study participants are presented in table 1 . The study population was comprised of 36 men and 36 women. Participants were middle-aged (age range 41.7066.30 years) (mean age for men and women $52.55 \pm 6.07$ and $56.19 \pm 6.13$ years, respectively), and all women were post-menopausal and not on hormone replacement therapy. Similarly to what we have reported before $[18,19]$, apnoeic women were slightly obese and tended to have a higher BMI compared with their respective controls $\left(30.39 \pm 3.37\right.$ versus $\left.27.95 \pm 4.27 \mathrm{~kg} \cdot \mathrm{m}^{-2} ; \mathrm{p}=0.07\right)$, while there was no BMI difference between apnoeic and control men $\left(26.94 \pm 2.58\right.$ versus $\left.26.42 \pm 2.61 \mathrm{~kg} \cdot \mathrm{m}^{-2} ; \mathrm{p}=0.55\right)$. As expected, sleep apnoeics slept worse than controls (table 1). Specifically, they demonstrated significantly increased wake time after sleep onset (WASO) and higher percentage of stage 1 sleep, while they spent less time in stage 2 sleep. Finally, patients with apnoea had significantly higher BDI-II scores, but their BAI scores were not significantly different. Similarly to what we have previously reported [18, 19], apnoeic women had significantly higher BDI-II scores than their controls, while no differences in BDI-II or BAI scores between apnoeic and control men were observed. However, it should be noted that due to our inclusion criteria, BDI-II scores were very low, and thus not indicative of clinical depression for both apnoeics and controls.

TABLE 1 Demographic, sleep, respiratory, anxiety and depression data in sleep apnoea patients versus controls at baseline

\begin{tabular}{|c|c|c|c|}
\hline & Controls & Apnoeics & p-value \\
\hline Subjects $n$ & 37 & 35 & \\
\hline Age years & $53.27 \pm 5.94$ & $55.54 \pm 6.59$ & 0.13 \\
\hline BMI $\mathrm{kg} \cdot \mathrm{m}^{-2}$ & $27.25 \pm 3.64$ & $28.52 \pm 3.40$ & 0.13 \\
\hline \multicolumn{4}{|c|}{ Blood pressure $\mathrm{mmHg}$} \\
\hline Systolic & $122.76 \pm 13.67$ & $128.26 \pm 15.43$ & 0.11 \\
\hline Diastolic & $74.95 \pm 7.81$ & $77.17 \pm 10.19$ & 0.30 \\
\hline Hypertension ${ }^{\#} \%$ & 18.9 & 31.4 & 0.17 \\
\hline AHI events $\cdot \mathrm{h}^{-1}$ & $2.30 \pm 1.92$ & $38.52 \pm 21.64$ & $<0.01$ \\
\hline Minimum $\mathrm{SaO}_{2}$ & $90.30 \pm 4.59$ & $82.06 \pm 6.62$ & $<0.01$ \\
\hline Sleep latency $\min$ & $16.05 \pm 10.47$ & $16.57 \pm 8.96$ & 0.82 \\
\hline WASO $\mathrm{min}$ & $58.84 \pm 33.20$ & $99.99 \pm 53.13$ & $<0.01$ \\
\hline TST min & $406.76 \pm 36.27$ & $364.29 \pm 53.85$ & $<0.01$ \\
\hline TST \% & $84.48 \pm 7.68$ & $75.78 \pm 11.32$ & $<0.01$ \\
\hline N1 \% & $12.53 \pm 4.50$ & $18.42 \pm 8.73$ & $<0.01$ \\
\hline N2 \% & $62.04 \pm 9.14$ & $54.92 \pm 12.49$ & $<0.01$ \\
\hline SWS $\%$ & $11.62 \pm 9.93$ & $12.72 \pm 10.17$ & 0.64 \\
\hline REM \% & $13.81 \pm 5.88$ & $13.94 \pm 6.86$ & 0.93 \\
\hline REM latency min & $115.87 \pm 65.38$ & $118.34 \pm 65.47$ & 0.87 \\
\hline BAl log & $0.38 \pm 0.40$ & $0.36 \pm 0.36$ & 0.85 \\
\hline BDI-II log & $0.39 \pm 0.38$ & $0.59 \pm 0.40$ & 0.03 \\
\hline
\end{tabular}

Data are presented as mean \pm SD, unless otherwise stated. BMI: body mass index; $\mathrm{AHI}$ : apnoea-hypopnea index; $\mathrm{SaO}_{2}$ : arterial oxygen saturation; WASO: wake after sleep onset; TST: total sleep time; N1: stage 1 sleep; N2: stage 2 sleep; SWS: slow wave sleep; REM: rapid eye movement; BAI: Beck Anxiety Inventory; BDI-II: Beck Depression Inventory II. " : hypertension defined as systolic blood pressure $\geqslant 140 \mathrm{mmHg}$ and/or diastolic blood pressure $\geqslant 90 \mathrm{mmHg}$. 
Cortisol profile in sleep apnoea patients versus controls

As there was no significant gender effect $(p=0.93)$, neither group-by-gender $(p=0.41)$ or gender-by-time $(\mathrm{p}=0.49)$ interaction effects, cortisol values between sleep apnoea patients and controls were compared for both genders together (table 2). Sleep apnoea patients had significantly higher cortisol levels during the entire 24-h period and during the night-time, whereas the difference became nonsignificant during the day (table 2).

There was no significant effect of OSA on the circadian secretory pattern of cortisol, as indicated by the absence of a significant group-by-time interaction effect $(\mathrm{p}>0.1)$. Specifically, in both control and apnoeic individuals, the morning peak cortisol secretion was observed between 07:00 and 08:00 h (figure 2). Also, no difference in cortisol nadir time occurrence or mean values was observed.

\section{Association of intermittent hypoxia (minimum $\mathrm{SaO}_{2}$ ) versus $\mathrm{AHI}$ versus sleep fragmentation with cortisol levels}

Using simple correlation analyses, average 24-h cortisol plasma levels had a trend correlation with WASO (as a marker of sleep fragmentation, $\mathrm{r}=0.23, \mathrm{p}=0.05$ ) and there was a statistical trend for night-time cortisol to be correlated with WASO $(r=0.20, p=0.09)$, but not $\log$ AHI $(r=0.18, p=0.15)$. However, no significant association was found with minimum $\mathrm{SaO}_{2}(\mathrm{r}=0.06 \mathrm{p}=0.60$ for average 24-h cortisol plasma levels and $\mathrm{r}=-0.03, \mathrm{p}=0.83$ for night-time cortisol). In a backward fully adjusted multiple regression model (where age, gender, BMI, anxiety, depression and smoking were forced in the model), average 24-h cortisol values had a trend correlation with $\log \mathrm{AHI}\left(\mathrm{r}^{2}=0.23, \beta=0.23, \mathrm{p}=0.08\right)$, whereas night-time cortisol was significantly associated with $\log \mathrm{AHI}\left(\mathrm{r}^{2}=0.19, \beta=0.36, \mathrm{p}=0.01\right)$.

\section{CPAP effect on cortisol circadian profile, blood pressure and emotional distress}

The average daily CPAP use was $6.04 \pm 1.19 \mathrm{~h}$, whereas the average daily sham-CPAP use was $5.17 \pm 1.22 \mathrm{~h}$. The majority of our participants, apart from two, were regular users. Given that sham-CPAP treatment administers a pressure as low as $1 \mathrm{cmH}_{2} \mathrm{O}$, it is possible that a subject could be able to tell the difference when assigned first to therapeutic CPAP treatment. However, only three subjects raised questions about the kind of treatment they were getting after the switch.

CPAP improved significantly the respiratory and sleep variables (table 3) and lowered 24-h cortisol levels (table 4) compared with baseline, while no significant difference from sham-CPAP was observed (table 4). However, there was a significant linear trend across the three phases, with the highest cortisol values observed at baseline, intermediate post-sham-CPAP and minimal post-CPAP $(\mathrm{p}<0.01)$. There was no significant effect in terms of the circadian secretory pattern of the hormone, as indicated by the absence of a significant group-by-time interaction effect $(\mathrm{p}>0.1)$. Specifically, in all phases, the morning peak cortisol secretion was observed between 07:00 and 08:00 h (figure 3), whereas the cortisol nadir time was similar across all phases (figure 3).

Finally, no significant effects of BMI $(p=0.86)$, anxiety $(p=0.28)$, depression $(p=0.30)$, order of the intervention $(\mathrm{p}=0.19)$ or apnoea severity $(\mathrm{p}=0.87)$ on cortisol values were observed.

TABLE 2 Cortisol levels in sleep apnoea patients versus controls at baseline

\begin{tabular}{lccc} 
& Controls & Apnoeics & p-value \\
\hline $\begin{array}{l}\text { Subjects } \\
\text { Model A }\end{array}$ & 37 & 35 & \\
$\quad$ Cortisol 24-h $\mu \mathrm{g} \cdot \mathrm{mL}^{-1}$ & $7.39 \pm 0.16$ & $7.87 \pm 0.17$ & 0.04 \\
$\quad$ Cortisol night-time $\mu \mathrm{g} \cdot \mathrm{mL}^{-1}$ & $7.10 \pm 0.21$ & $7.99 \pm 0.22$ & $<0.01$ \\
$\quad$ Cortisol daytime $\mu \mathrm{g} \cdot \mathrm{mL}^{-1}$ & $7.56 \pm 0.23$ & $7.80 \pm 0.23$ & 0.48 \\
Model B & $7.23 \pm 0.17$ & $8.06 \pm 0.17$ & $<0.01$ \\
$\quad$ Cortisol 24-h $\mu \mathrm{g} \cdot \mathrm{mL}^{-1}$ & $6.85 \pm 0.21$ & $8.29 \pm 0.24$ & $<0.01$ \\
$\quad$ Cortisol night-time $\mu \mathrm{g} \cdot \mathrm{mL}^{-1}$ & $7.46 \pm 0.24$ & $7.91 \pm 0.23$ & 0.20 \\
$\quad$ Cortisol daytime $\mu \mathrm{g} \cdot \mathrm{mL}^{-1}$ & & & \\
\hline
\end{tabular}

Data are presented as $\mathrm{n}$ or estimated mean \pm SE after adjustment for the following factors. Model A: unadjusted values, and fixed factors added in the model group and group-by-time. Model B: fixed factors in the model for cortisol: group (controls versus apnoeics), gender and time as well as their interactions, age, body mass index, anxiety (log Beck Anxiety Inventory), depression (log Beck Depression Inventory II), and smoking. 


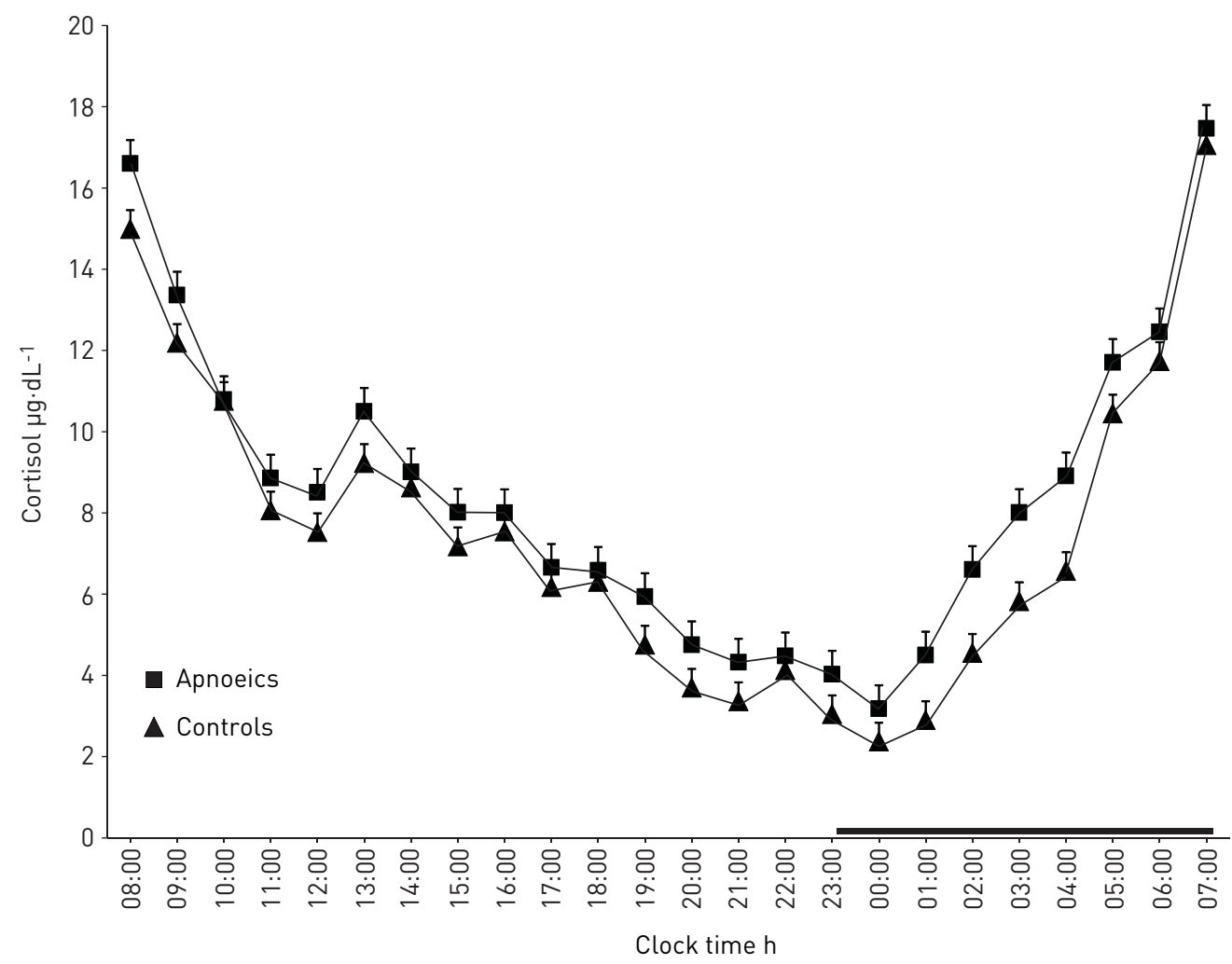

FIGURE 2 Serial 24-h cortisol values at baseline in apnoeics versus controls. The thick black line on the abscissa indicates the night-time sleep recording period.

There was a slight but nonsignificant decrease of systolic and diastolic blood pressure (table 4). Blood pressure results did not change when analyses were repeated by excluding those receiving antihypertensive medications (results not shown).

Finally, CPAP did not decrease BDI-II scores compared with baseline and had no significant effect on BAI scores (table 4). As aponeic women had higher BDI-II scores compared with their respective controls, but still not indicative of clinical depression, we also examined whether CPAP would have stronger effect on depression scores in women rather than in men. Analysis by gender did not reveal any significant differences on BDI-II scores after the use of CPAP in men or women separately.

TABLE 3 Sleep data in sleep apnoea patients at baseline, post-continuous positive airway pressure (CPAP) and post-sham-CPAP

\begin{tabular}{lcccccc} 
& Baseline & CPAP & Sham-CPAP & p1-value & p2-value & p3-value \\
\hline Subjects & 35 & 35 & 35 & & & \\
Sleep latency min & $16.57 \pm 1.52$ & $14.28 \pm 1.83$ & $14.87 \pm 2.21$ & 1.00 & 1.00 & 1.00 \\
WAS0 min & $99.99 \pm 8.98$ & $70.26 \pm 7.56$ & $91.43 \pm 8.77$ & 0.04 & 1.00 & 0.22 \\
TST min & $364.29 \pm 9.10$ & $395.32 \pm 8.37$ & $373.02 \pm 9.17$ & 0.04 & 1.00 & 0.23 \\
TST \% & $75.78 \pm 1.91$ & $82.35 \pm 1.69$ & $72.92 \pm 1.91$ & 0.04 & 1.00 & 0.26 \\
N1 \% & $18.42 \pm 1.47$ & $12.54 \pm 0.93$ & $17.00 \pm 1.37$ & $<0.01$ & 1.00 & 0.03 \\
N2 \% & $54.92 \pm 2.11$ & $59.22 \pm 1.87$ & $58.93 \pm 1.88$ & 0.40 & 0.48 & 1.00 \\
SWS \% & $12.72 \pm 1.72$ & $12.37 \pm 1.79$ & $11.83 \pm 1.67$ & 1.00 & 1.00 & 1.00 \\
REM \% & $13.94 \pm 1.16$ & $14.15 \pm 0.97$ & $12.23 \pm 1.04$ & 1.00 & 0.83 & 0.55 \\
REM latency min & $118.34 \pm 11.07$ & $122.12 \pm 12.79$ & $137.87 \pm 11.34$ & 1.00 & 0.67 & 1.00 \\
\hline
\end{tabular}

Data are presented as $\mathrm{n}$ or mean士SE. WASO: wake after sleep onset; TST: total sleep time; N1: stage 1 sleep; N2: stage 2 sleep; SWS: slow wave sleep; REM: rapid eye movement; p1: comparison between baseline and CPAP; p2: comparison between baseline and sham-CPAP; p3: comparison between CPAP and sham-CPAP. 
TABLE 4 Cortisol levels in sleep apnoea patients at baseline, post-continuous positive airway pressure (CPAP) and post-sham-CPAP

\begin{tabular}{|c|c|c|c|c|c|c|}
\hline & Baseline & CPAP & Sham-CPAP & p1-value & p2-value & p3-value \\
\hline Subjects & 35 & 35 & 35 & & & \\
\hline BMI $\mathrm{kg} \cdot \mathrm{m}^{-2}$ & $28.52 \pm 0.57$ & $29.18 \pm 0.60$ & $28.81 \pm 0.59$ & 1.00 & 1.00 & 1.00 \\
\hline AHI events $\cdot h^{-1}$ & $38.52 \pm 3.73$ & $2.58 \pm 0.75$ & $32.41 \pm 4.92$ & $<0.01$ & 0.97 & $<0.01$ \\
\hline Minimum $\mathrm{SaO}_{2}$ & $82.06 \pm 1.12$ & $91.57 \pm 0.75$ & $83.17 \pm 1.16$ & $<0.01$ & 1.00 & $<0.01$ \\
\hline \multicolumn{7}{|l|}{ Blood pressure $\mathrm{mmHg}$} \\
\hline Systolic & $128.26 \pm 2.61$ & $126.17 \pm 2.79$ & $121.77 \pm 2.47$ & 1.00 & 0.22 & 0.73 \\
\hline Diastolic & $77.37 \pm 1.76$ & $75.17 \pm 1.64$ & $74.14 \pm 1.59$ & 1.00 & 0.53 & 1.00 \\
\hline BAI log & $0.36 \pm 0.06$ & $0.27 \pm 0.06$ & $0.35 \pm 0.06$ & 0.89 & 1.00 & 1.00 \\
\hline BDI-II log & $0.59 \pm 0.06$ & $0.46 \pm 0.06$ & $0.67 \pm 0.06$ & 0.32 & 0.65 & $<0.01$ \\
\hline Cortisol $24-\mathrm{h} \mu \mathrm{g} \cdot \mathrm{mL}^{-1}$ & $7.89 \pm 0.15$ & $7.25 \pm 0.14$ & $7.57 \pm 0.19$ & $<0.01$ & 0.57 & 0.57 \\
\hline Cortisol night-time $\mu \mathrm{g} \cdot \mathrm{mL}^{-1}$ & $8.01 \pm 0.24$ & $7.35 \pm 0.21$ & $7.68 \pm 0.28$ & 0.14 & 1.00 & 1.00 \\
\hline Cortisol daytime $\mu \mathrm{g} \cdot \mathrm{mL}^{-1}$ & $7.82 \pm 0.20$ & $7.18 \pm 0.19$ & $7.49 \pm 0.26$ & 0.07 & 0.96 & 1.00 \\
\hline
\end{tabular}

Data are presented as $\mathrm{n}$ or estimated mean \pm SE after adjustment for the following factors. Fixed factors in the final models for cortisol: group (baseline, sham-CPAP, CPAP), gender and time as well as their interactions, body mass index (BMI), anxiety, depression, order of intervention (sham-CPAP first or CPAP first), and apnoea severity (mild/moderate versus severe). $\mathrm{AHI}$ : apnoea-hypopnea index; $\mathrm{SaO}_{2}$ : arterial oxygen saturation; BAI: Beck Anxiety Inventory; BDI-II: Beck Depression Inventory II; p1: comparison between baseline and CPAP; p2: comparison between baseline and sham-CPAP; p3: comparison between CPAP and sham-CPAP.

\section{Discussion}

The primary findings of this study are: 1) sleep apnoea in middle-aged nonobese men and slightly obese women is associated with significantly higher 24-h cortisol levels compared with age- and BMI-matched controls, and 2) CPAP appears to lower significantly the baseline HPA axis activity.

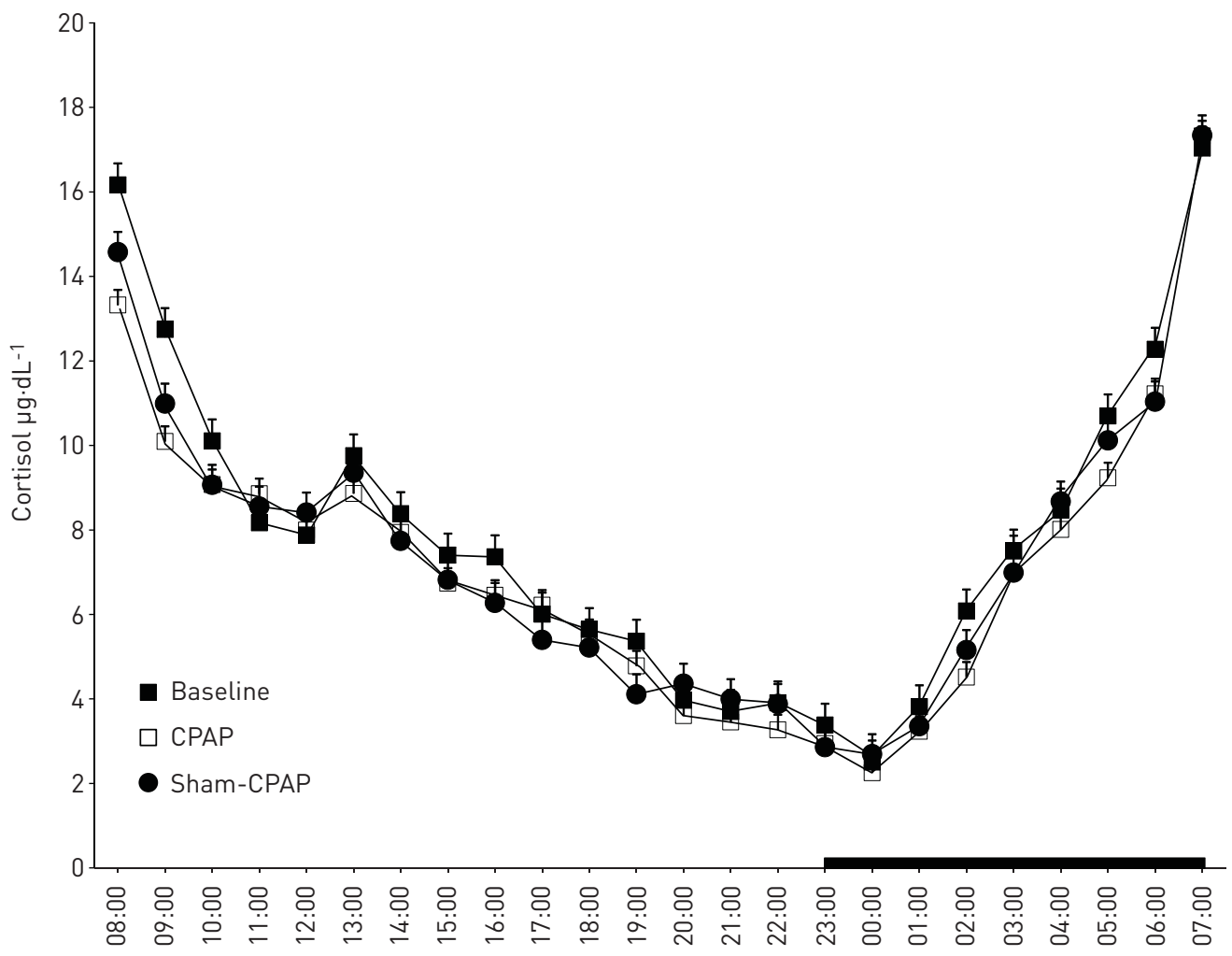

Clock time h

FIGURE 3 Serial 24-h cortisol values at baseline versus continuous positive airway pressure (CPAP) versus sham-CPAP. The thick black line on the abscissa indicates the night-time sleep recording period. 
In a previous study using similar methodology in a group of obese men with sleep apnoea, we demonstrated a small but significant nocturnal elevation of cortisol levels compared with BMI- and age-matched controls [11]. These levels returned to normal by CPAP use. In this study, we demonstrated a similar albeit stronger difference of HPA axis activity between nonobese men with sleep apnoea and their matched controls. Specifically, this difference was significant for the entire 24-h plasma cortisol levels and became highest at night-time, as this is the period when the apnoeic events occur. The difference was not only statistically significant, but also clinically relevant as evidenced by an increase of cortisol levels by 15-20\% compared with controls. Such a difference in a chronic basis is considered to be associated with adverse cardiometabolic effects such as hypertension, type 2 diabetes, as well as osteoporosis and others [20]. That our results were stronger in nonobese men compared with obese men can be explained by the modifying effect of obesity on the HPA axis. Specifically, we have shown that obesity is associated with lower cortisol values compared with normal-weight controls [11], which might have a masking effect on the association of apnoea and HPA axis in obese subjects.

Our study is the first to show that women, similarly to men, suffer from the same degree of the HPA axis activation as a result of the intermittent hypoxia and sleep fragmentation associated with this sleep disorder. We have previously suggested that women with apnoea tend to be more obese than men, but without evidence of visceral adiposity, whereas their inflammation profile appears to be less severe compared with men $[18,19,21]$. We did not detect any sexual dimorphic effects in terms of sleep apnoea-associated HPA axis activation. Given the association of hypercortisolaemia with cardiovascular problems such as hypertension, this may explain why men and women with apnoea are at similar risk for this disorder. Future studies should examine whether the milder inflammation metabolic profile in apnoeic women may affect gender-wise medical morbidity, e.g. myocardial infarction, stroke or longevity.

In our study, participants with clinical depression were excluded. However, apnoeic women scored significantly higher on depression scales compared with their respective controls, while no such difference was observed in men. This finding is consistent with previous studies reporting that apnoea is associated with depression in women more commonly than in men $[22,23]$ and suggests that women may be more vulnerable to the effects of chronic hypercortisolaemia, a risk factor for depression. Some of the studies (but not all), on primarily male samples, have reported that OSA is associated with depression relieved by CPAP use [24-28], with female gender being a risk factor for persistent symptoms [25].

Similarly to our previous study in obese men, CPAP had a significant effect on cortisol levels in nonobese men and slightly obese women compared with baseline. This effect could not be explained by the expectations raised by the administration of the treatment alone. This finding is consistent with the reported immediate effects of CPAP, e.g. on blood pressure recordings or the relief to some extent provided by CPAP for excessive daytime sleepiness and fatigue [29-31]. The fact that in our study the effect on blood pressure was not significant may be the result of a relatively small sample and increased variance within our subjects.

Finally, we should note that the beneficial effects of CPAP appear to be partial as previous studies have shown that CPAP does not, in short-term use, affect the inflammation/metabolic profile of patients with sleep apnoea [19, 32,33]. This is important given the independent association of insulin resistance and inflammation with cardiovascular morbidity and mortality.

A significant limitation of our study is our inability to match apnoeic and control women in terms of BMI. However, the weight of our women participants was at the lower end of the BMI of women with OSA typically evaluated in the sleep disorder clinic or those detected in epidemiological samples. Furthermore, the higher BMI of apnoeic women versus controls might have biased our findings in the direction of masking any difference as obesity is associated with lower basal cortisol levels. This increases our confidence that the findings of our study reflect the true difference of cortisol levels between women with apnoea and controls. Another limitation is that sham-CPAP is not associated with $100 \%$ blindness, as indicated in our study by the three participants that were able to recognise the switch in treatment. However, although sham-CPAP does not provide an exact replication of the therapeutic CPAP (like placebo in cases of medication trials), it is the only and best available placebo treatment in CPAP placebo-controlled trials.

In conclusion, sleep apnoea, in both obese and nonobese men, is associated with activation of the HPA axis compared with controls and this difference is stronger in nonobese individuals. Also, women suffer from the same degree of HPA axis activation, which may explain why both genders are affected similarly, e.g. by hypertension. Finally, CPAP appears to reverse hypercortisolaemia, which may in turn explain the beneficial effect of CPAP on cardiovascular morbidity, i.e. hypertension.

\section{Acknowledgements}

The authors would like to acknowledge Dr Hung-Mo Lin (Research Professor, Mount Sinai Hospital, New York, NY, USA) for her significant contribution to the methodology and statistical analysis. 


\section{References}

$1 \quad$ Vgontzas AN, Kales A. Sleep and its disorders. Annu Rev Med 1999; 50: 387-400.

2 Späth-Schwalbe E, Gofferje M, Kern W, et al. Sleep disruption alters nocturnal ACTH and cortisol secretory patterns. Biol Psychiatry 1991; 29: 575-584.

3 Lanfranco F, Gianotti L, Pivetti S, et al. Obese patients with obstructive sleep apnoea syndrome show a peculiar alteration of the corticotroph but not of the thyrotroph and lactotroph function. Clin Endocrinol (Oxf) 2004; 60: $41-48$.

4 Panaree B, Chantana M, Wasana S, et al. Effects of obstructive sleep apnea on serum brain-derived neurotrophic factor protein, cortisol, and lipid levels. Sleep Breath 2011; 15: 649-656.

5 Lam JC, Yan CS, Lai AY, et al. Determinants of daytime blood pressure in relation to obstructive sleep apnea in men. Lung 2009; 187: 291-298.

6 Barceló A, Barbé F, de la Peña M, et al. Insulin resistance and daytime sleepiness in patients with sleep apnoea. Thorax 2008; 63: 946-950.

7 Grunstein RR, Handelsman DJ, Lawrence SJ, et al. Neuroendocrine dysfunction in sleep apnea: reversal by continuous positive airways pressure therapy. J Clin Endocrinol Metab 1989; 68: 352-358.

8 Nakamura T, Chin K, Shimizu K, et al. Acute effect of nasal continuous positive airway pressure therapy on the systemic immunity of patients with obstructive sleep apnea syndrome. Sleep 2001; 24: 545-553.

9 Karaca Z, Ismailogullari S, Korkmaz S, et al. Obstructive sleep apnoea syndrome is associated with relative hypocortisolemia and decreased hypothalamo-pituitary-adrenal axis response to 1 and $250 \mu \mathrm{g} \mathrm{ACTH}$ and glucagon stimulation tests. Sleep Med 2013; 14: 160-164.

10 Bratel T, Wennlund A, Carlström K. Pituitary reactivity, androgens and catecholamines in obstructive sleep apnoea. Effects of continuous positive airway pressure treatment (CPAP). Respir Med 1999; 93: 1-7.

11 Vgontzas AN, Pejovic S, Zoumakis E, et al. Hypothalamic-pituitary-adrenal axis activity in obese men with and without sleep apnea: effects of continuous positive airway pressure therapy. J Clin Endocrinol Metab 2007; 92: 4199-4207.

12 Edwards KM, Kamat R, Tomfohr LM, et al. Obstructive sleep apnea and neurocognitive performance: the role of cortisol. Sleep Med 2014; 15: 27-32.

13 Tomfohr LM, Edwards KM, Dimsdale JE. Is obstructive sleep apnea associated with cortisol levels? A systematic review of the research evidence. Sleep Med Rev 2012; 16: 243-249.

14 Carneiro G, Togeiro SM, Hayashi LF, et al. Effect of continuous positive airway pressure therapy on hypothalamic-pituitary-adrenal axis function and 24-h blood pressure profile in obese men with obstructive sleep apnea syndrome. Am J Physiol Endocrinol Metab 2008; 295: E380-E384.

15 Henley DE, Russell GM, Douthwaite JA, et al. Hypothalamic-pituitary-adrenal axis activation in obstructive sleep apnea: the effect of continuous positive airway pressure therapy. J Clin Endocrinol Metab 2009; 94: 4234-4242.

16 Meston N, Davies RJ, Mullins R, et al. Endocrine effects of nasal continuous positive airway pressure in male patients with obstructive sleep apnoea. J Intern Med 2003; 254: 447-454.

17 Schmoller A, Eberhardt F, Jauch-Chara K, et al. Continuous positive airway pressure therapy decreases evening cortisol concentrations in patients with severe obstructive sleep apnea. Metabolism 2009; 58: 848-853.

18 Kritikou I, Basta M, Tappouni R, et al. Sleep apnoea and visceral adiposity in middle-aged male and female subjects. Eur Respir J 2013; 41: 601-609.

19 Kritikou I, Basta M, Vgontzas AN, et al. Sleep apnea, sleepiness, inflammation and insulin resistance in middle-aged men and women. Eur Respir J 2014; 43: 145-155.

20 Chrousos GP. Stress and disorders of the stress system. Nat Rev Endocrinol 2009; 5: 374-381.

21 Gaines J, Vgontzas AN, Fernandez-Mendoza J, et al. Gender differences in the association of sleep apnea and inflammation. Brain Behav Immun 2015; 47: 211-217.

22 Basta M, Lin HM, Pejovic S, et al. Lack of regular exercise, depression, and degree of apnea are predictors of excessive daytime sleepiness in patients with sleep apnea: sex differences. J Clin Sleep Med 2008; 4: 19-25.

23 Quintana-Gallego E, Carmona-Bernal C, Capote F, et al. Gender differences in obstructive sleep apnea syndrome: a clinical study of 1166 patients. Respir Med 2004; 98: 984-989.

24 Habukawa M, Uchimura N, Kakuma $\mathrm{T}$, et al. Effect of CPAP treatment on residual depressive symptoms in patients with major depression and coexisting sleep apnea: contribution of daytime sleepiness to residual depressive symptoms. Sleep Med 2010; 11: 552-557.

25 Gagnadoux F, Le Vaillant M, Goupil F, et al. Depressive symptoms before and after long-term CPAP therapy in patients with sleep apnea. Chest 2014; 145: 1025-1031

26 Means MK, Lichstein KL, Edinger JD, et al. Changes in depressive symptoms after continuous positive airway pressure treatment for obstructive sleep apnea. Sleep Breath 2003; 7: 31-42.

27 Schwartz DJ, Kohler WC, Karatinos G. Symptoms of depression in individuals with obstructive sleep apnea may be amenable to treatment with continuous positive airway pressure. Chest 2005; 128: 1304-1309.

28 Ye L, Pien GW, Ratcliffe SJ, et al. Gender differences in obstructive sleep apnea and treatment response to continuous positive airway pressure. J Clin Sleep Med 2009; 5: 512-518.

29 Alajmi M, Mulgrew AT, Fox J, et al. Impact of continuous positive airway pressure therapy on blood pressure in patients with obstructive sleep apnea hypopnea: a meta-analysis of randomized controlled trials. Lung 2007; 185: $67-72$.

30 Montesi SB, Edwards BA, Malhotra A, et al. The effect of continuous positive airway pressure treatment on blood pressure: a systematic review and meta-analysis of randomized controlled trials. J Clin Sleep Med 2012; 8: 587-596.

31 Pépin JL, Tamisier R, Barone-Rochette G, et al. Comparison of continuous positive airway pressure and valsartan in hypertensive patients with sleep apnea. Am J Respir Crit Care Med 2010; 182: 954-960.

32 Vgontzas AN, Zoumakis E, Bixler EO, et al. Selective effects of CPAP on sleep apnoea-associated manifestations. Eur J Clin Invest 2008; 38: 585-595.

33 Kohler M, Ayers L, Pepperell JC, et al. Effects of continuous positive airway pressure on systemic inflammation in patients with moderate to severe obstructive sleep apnoea: a randomised controlled trial. Thorax 2009; 64: 67-73. 